\title{
Towards fully automated Identification of Vesicle-Membrane Fusion Events in TIRF Microscopy
}

\author{
Pascal Vallotton $^{1 *}$, David E. James ${ }^{2}$, and William E. Hughes ${ }^{2}$ \\ ${ }^{l}$ CSIRO, Mathematical and Information Sciences, Locked Bag 17, North Ryde, NSW 1670, Australia \\ ${ }^{2}$ The Garvan Institute of Medical Research, University of New South Wales, 384 Victoria St, NSW \\ 2010, Australia \\ * Correspondence to: Pascal Vallotton, Biotech Imaging, CSIRO Mathematical and Information \\ Sciences, Locked Bag 17, North Ryde, NSW 1670, Australia \\ Phone: +61 (0)293253208 \\ Fax: $+61(0) 293253200$ \\ E-mail: pascal.vallotton@csiro.au
}

\begin{abstract}
Total Internal Reflection Fluorescence Microscopy (TIRFM) is imposing itself as the tool of choice for studying biological activity in close proximity to the plasma membrane. For example, the exquisite selectivity of TIRFM allows monitoring the diffusion of GFP-phogrin vesicles and their recruitment to the plasma membrane in pancreatic $\beta$-cells. We present a novel computer vision system for automatically identifying the elusive fusion events of GFP-phogrin vesicles with the plasma membrane. Our method is based on robust object tracking and matched filtering. It should accelerate the quantification of TIRFM data and allow the extraction of more biological information from image data to support research in diabetes and obesity.
\end{abstract}

Keywords: Vesicles, vesicle tracking, vesicle fusion, insulin regulation, GFP-phogrin

\section{INTRODUCTION}

The transfer of material between intracellular organelles is mediated by membrane vesicles that continuously bud from donor organelles and fuse with target membranes. The same process can control the transfer of materials coming into or being secreted out of a cell. Thus knowledge of the mechanisms allowing and controlling vesicle trafficking events are essential for an understanding of basic cell function but also for understanding the way cells behave and perform in the context of a multicellular organism.

Maintenance of glucose homeostasis in mammalian organisms requires accurate regulation of insulin secretion from pancreatic $\beta$-cells in response to a variety of nutrient, hormonal and neurological factors. Coordination of these inputs through signal transduction pathways results in the controlled translocation of insulin containing vesicles from their sites of synthesis (ER and Golgi apparatus) to the 
plasma membrane, where regulated docking and fusion with the membrane control secretion of insulin. Although a failure of insulin secretion is the defining feature of Type 2 Diabetes, the precise site of this failure is unclear. A better understanding of the mechanisms underlying insulin secretion is therefore essential, not only to identify the site of the lesion in diabetes, but also to develop therapeutics to overcome it.

The incidence of Type 2 diabetes mellitus, with its associated morbidity and mortality, is rapidly increasing and is predicted to affect 1.3 million Australians by 2010 (Diabetes Australia).

GFP-phogrin has been widely used to monitor vesicular dynamics in pancreatic $\beta$ cells. This molecule is targeted to insulin containing vesicles where it is expressed as a transmembrane protein.

Upon glucose stimulation of pancreatic $\beta$-cells expressing GFP-phogrin, puffs of fluorescence can be observed under Total Internal Reflection Fluorescence Microscopy (TIRFM), indicating fusogen activity. Puffs display a biphasic evolution. First a transient and moderate increase of fluorescence is observed, as the fluorophores come in close proximity to the cover slip. In a second phase, diffusion of the fluorophores away from the fusion site causes a rapid decrease in intensity and a spreading of the signal (see Fig. 5). The observation of these events critically depends on the ability of TIRFM to image only a layer extending approximately $100 \mathrm{~nm}$ into the sample; the signal intensity for a given probe decreasing exponentially away from the cover slip (1).

Typical TIRFM movies contain several thousand image frames, where hundreds of vesicles can be seen to diffuse randomly, to dock, undock and to fuse. The attention of the visual system is attracted to all of this activity. As a result, it is extremely difficult to focus on one or the other aspect; let alone attempt to quantify it.

The present paper focuses on quantifying fusion activity as a key mechanism of insulin regulation. In order to identify fusion events, we combine object tracking with matched filters designed empirically.

Our system is not the first to facilitate annotation of TIRFM data. For example, Huang et al. report a semi-automated system that allowed to speed up the process (2). Tran et al. also develop quantitative techniques to reveal the existence of a pool of fusion-ready vesicles, 200-300 nm away from the plasma membrane (3). Here, we are putting the effort on the long term goal of making TIRFM amenable to high throughput analysis, which will demand a much greater level of automation.

Our results provide initial evidence that it is possible to detect a great majority of events automatically and that the rate of false positive and missed events compares favorably to what a human operator can achieve, yet at a small fraction of the time. 


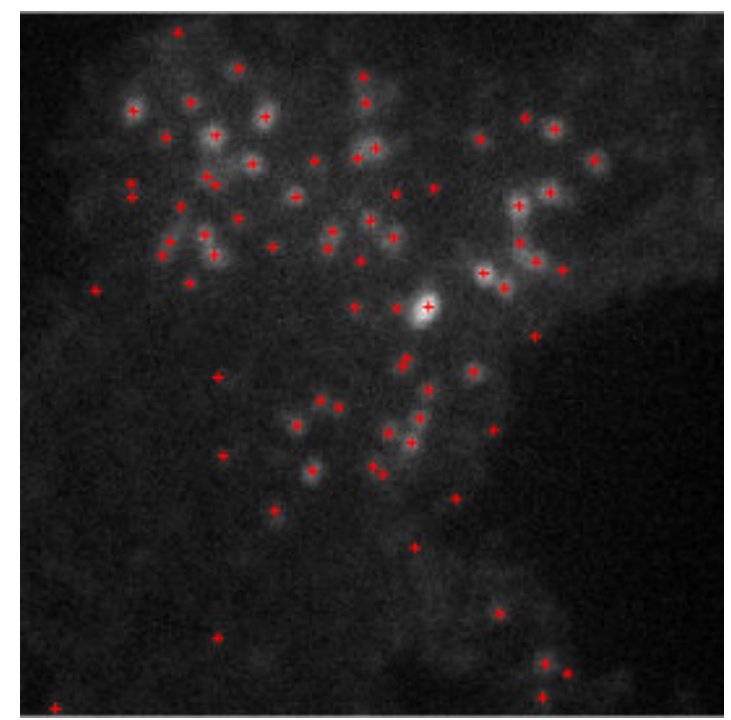

FIGURE 1. First frame of a movie of diffusing GFP-phogrin vesicles seen under TIRFM. Red crosses identify the positions of vesicles.

\section{MATERIAL AND METHODS AND RESULTS}

\section{Cell preparation and image acquisition}

MIN6 pancreatic beta cells grown in high glucose $(25 \mathrm{mM})$ Dulbecco's modified Eagle Medium (Invitrogen) supplemented with 10\% foetal calf serum (Trace, Melbourne, Australia), $15 \mathrm{mM}$ HEPES, $50 \mathrm{IU} / \mathrm{ml}$ penicillin and $50 \mu \mathrm{g} / \mathrm{ml}$ streptomycin (Invitrogen) were seeded onto $42 \mathrm{~mm}$ No. 1 glass coverslips and transiently transfected via lipofectamine 2000 (Invitrogen) with DNA encoding an $\mathrm{NH}_{2}$-terminally truncated and GFP-tagged derivative of the insulin-vesicle-resident protein 'phogrin' (phogrin-his633-eGFP). 24-48 hours after transfection, coverslips were washed in $37^{\circ} \mathrm{C} \mathrm{KRB}$ buffer (Modified Krebs-Ringer bicarbonate buffer: 136 $\mathrm{mM} \mathrm{NaCl}, 4.7 \mathrm{mM} \mathrm{KCl}, 5 \mathrm{mM} \mathrm{NaHCO} 3,1.2 \mathrm{mM} \mathrm{MgSO}_{4}\left(7 \mathrm{H}_{2} \mathrm{O}\right), 1.2 \mathrm{mM} \mathrm{KH}_{2} \mathrm{PO}_{4}$, $1 \mathrm{mM} \mathrm{CaCl}_{2}, 10 \mathrm{mM}$ HEPES (pH 7.4), glucose $2.8 \mathrm{mM}$, bubbled with $\sim 5 \% \mathrm{CO}_{2}$ for 15 minutes at $\mathrm{pH} 7.4$ confirmed, and bovine serum albumin was added to a final concentration of $5 \% \mathrm{w} / \mathrm{v}$. After approximately 30 minutes, the coverslip was mounted in a pre-warmed perfusion chamber POC-R (Pecon) and the underside cleaned with warm water and lint free tissues. The un-covered chambered coverslip was mounted in a heated stage microscope insert ' $\mathrm{P}$ ' (Pecon) on a Axiovert 200M (Zeiss) equipped with a large incubator (XL, Pecon) maintained at $37^{\circ} \mathrm{C}$. Suitably transfected GFPexpressing cells were identified by fluorescence using a 100x objective (NA 1.4 Alpha-Plan, Zeiss) and total internal reflection fluorescence microscopy (TIRFM) was performed using a $488 \mathrm{~nm}$ laser introduced into the excitation light path $(488 / 5 \mathrm{~nm})$ through the TIRF-slider (Zeiss) and appropriately angled to image approximately 150 $\mathrm{nm}$ into cells. Fluorescence $(525 / 25 \mathrm{~nm})$ was detected using an AxioCamMRm (Zeiss) and images were taken at a rate of 2 frames/second. Cells were treated with glucose 
(to a final concentration of $25 \mathrm{nM}$ ) as potent secretagogue inducing rapid release of insulin from internal vesicles in pancreatic beta cells (4).

\section{Image analysis}

We reasoned that fusion events are associated with the termination of vesicle trajectories (also called "death events"). A vesicle trajectory corresponds to the set of positions successively occupied by the same vesicle. We acquired images at a rate of 2 per second, such that vesicles did not have time to move a lot from one frame to the next. By inspection, the maximum amplitude of vesicle jumps that we observed was on the order of 7 pixels. Upon fusion of a vesicle with the cell membrane, the fluorescence of the vesicle rapidly diffuses into the background such that it is not possible to continue the trajectory.

Importantly, trajectories may also end because a vesicle simply undocks from the plasma membrane, or because two vesicles fuse together, or because the trajectory was built erroneously. Therefore, one needs additional cues for flagging a fusion event with confidence. As described in the introduction, a fusion event is characterized by a distinctive spatiotemporal evolution of the signal intensity. One hopes to be able to probe the complete movie for fusion events by convolution with this signature. We now describe the process of identifying death events, and of building and using a matched filter in more detail.

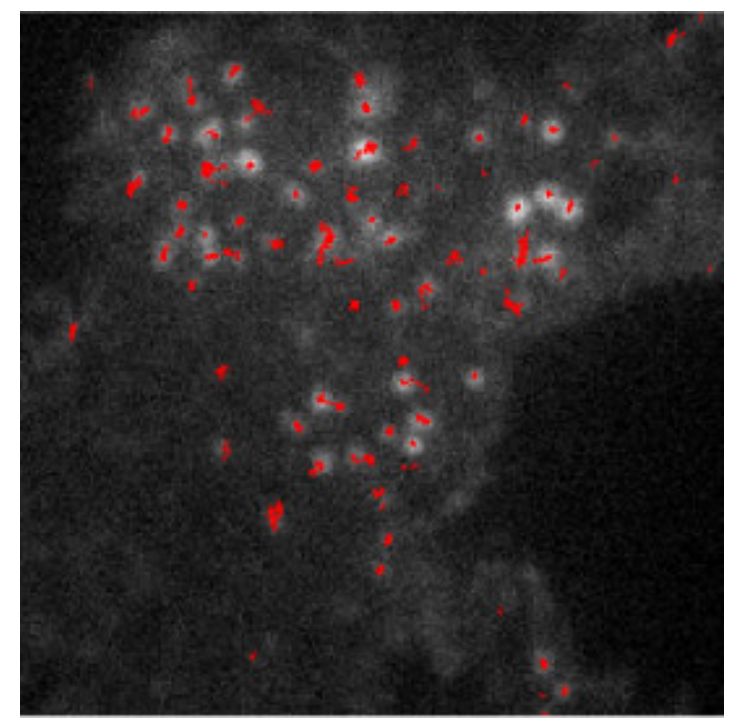

FIGURE 2. Trajectories lasting for more than 5 image frames have been produced automatically and are shown, overlaid on the first image of the movie.

\section{Identifying trajectory endings}

Building trajectories manually would be even more time consuming than identifying fusion events by inspection. Therefore, we built trajectories automatically 
using a commercially available object tracking software (Diatrack, Chavannes, Switzerland). The inference engine of the software allows tracking a variable number of particles, which was important for the present application. The software relies on the max-flow min-cost graph algorithm to produce an optimal assignment of vesicles identified in one image frame with those of the next frame (5).

The software was sensitive enough to identify even low contrast vesicles (see Fig. 1). Identifying low contrast vesicles is important as otherwise, a large number of trajectories would break when the contrast of a vesicle diminishes temporarily (false positive event). However, detection of low contrast features is sometimes due to spurious effects, including image sensor noise. Therefore, after producing all particle trajectories, we only kept those that lasted for more than 5 consecutive frames. The resulting tracks are shown in Figure 2. Simultaneous occurrence of a particle for 5 consecutive frames represents very solid evidence that the track really corresponds to a vesicle. A fusion event itself lasts on the order of 5 frames so it is very unlikely that this practice can lead to ignoring some events. This can only be verified at the end of the procedure, by inspection.

We then obtained the positions: $\mathrm{x}, \mathrm{y}$, and time: $\mathrm{t}$ of every track endings. This corresponded to a total set of 130 candidate fusion events for a movie of 100 frames. It is still cumbersome for a human operator to visually validate so many events. Additional automation is required.

A false negative event corresponds to a track that was continued whereas in reality, a fusion event occurred. This can happen only in the exceptional situation where a track was continued because a new particle appeared in the immediate vicinity of the fusion event, and in the very next frame. As mentioned, the maximum jump that we observed for the normal diffusive behavior of vesicles in our sequence was equal to 7 pixels. The tracking software is typically provided with this information from the user or determines it by itself. It will not build any trajectory that contains a jump larger than the specified value. Accordingly, the probability of a false negative event is extremely low, as the new particle would have to appear within an area of radius 7 pixels around the death. The number of genuine fusions in our movie, as obtained by manual examination was four, corresponding to 0.04 fusion per frame, on average. By examination of the movie, an upper limit on the number of particle appearance per frame was determined to be 0.2 . A disk of radius 7 pixels represented a fraction equal to $5 \mathrm{e}-4$ of the total area. Consequently, the probability for a false negative event per frame is approximately $4 \mathrm{e}-6$.

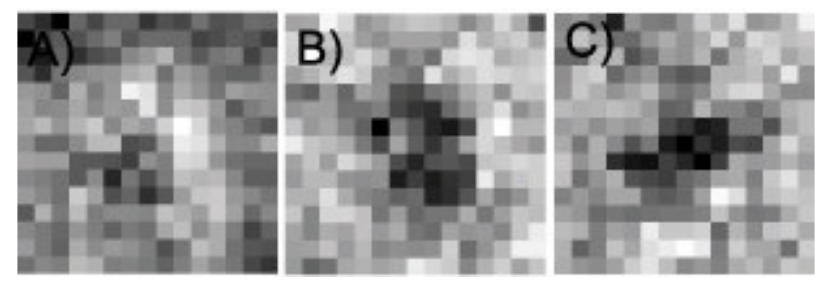

FIGURE 3. The three slices of the correlation kernel are shown. Immediately after fusion, the fluorescence intensity decreases most at the centre of the vesicle. 


\section{Matched filtering}

As there are 130 potential events identified automatically and only 4 genuine fusion events in our movie, the probability per frame of a false positive event is very high. We address this problem by probing the evolution of the fluorescence intensity around the trajectory endings and examine whether it complies with the typical signature of a fusion event. This can be done using a variety of distance measures, including the sum of squares difference, or the correlation. We used correlation with a $3 \mathrm{D}$ window of size $14 \times 14 \times 3$ around the most striking fusion event that we could visually identify (see Figure 5, row 5). Initial attempts in the direct space were not successful because of the very significant intensity differences between different fusion events.

Building the filter kernel for the data set corresponding to the set of difference images turned out to be more robust:

$$
\overline{\mathbf{I}}_{\mathbf{k}}=\mathbf{I}_{\mathbf{k}+1}-\mathbf{I}_{\mathbf{k}}, \mathbf{k}=1: \mathbf{N}-1,
$$

where $\mathrm{N}$ is the total number of image frames in the movie. The 3 slices of the correlation kernel in this difference of image space are shown in Figure 3. Note the typical dip that develops in the kernel centre as the fluorescence intensity decreases most in that area from one image to the next. The correlation kernel is not rotationally symmetric and one may fear that this could jeopardize the capacity of the algorithm to generalize. In fact, it will be demonstrated that our method is able to identify all fusion events in this particular movie.

The transformed movie of 99 frames, considered as single data set was correlated with the kernel. Specifically, the Pearson correlation coefficient on a pixel by pixel basis between the correlation kernel and each test region was calculated using the command IMFILTER( ( $\overline{\mathbf{I}}$, kernel, 'corr') in Matlab. In order to visualize the result, we generated iso-surfaces in MatlabTM with a threshold equal to 1/3 of the maximum correlation value observed. The result is shown in Figure 4, where the location corresponding to the template fusion event is indicated. Clearly, the best correlation value is obtained on the location of the template itself but many other locations qualify as well at the selected threshold. 


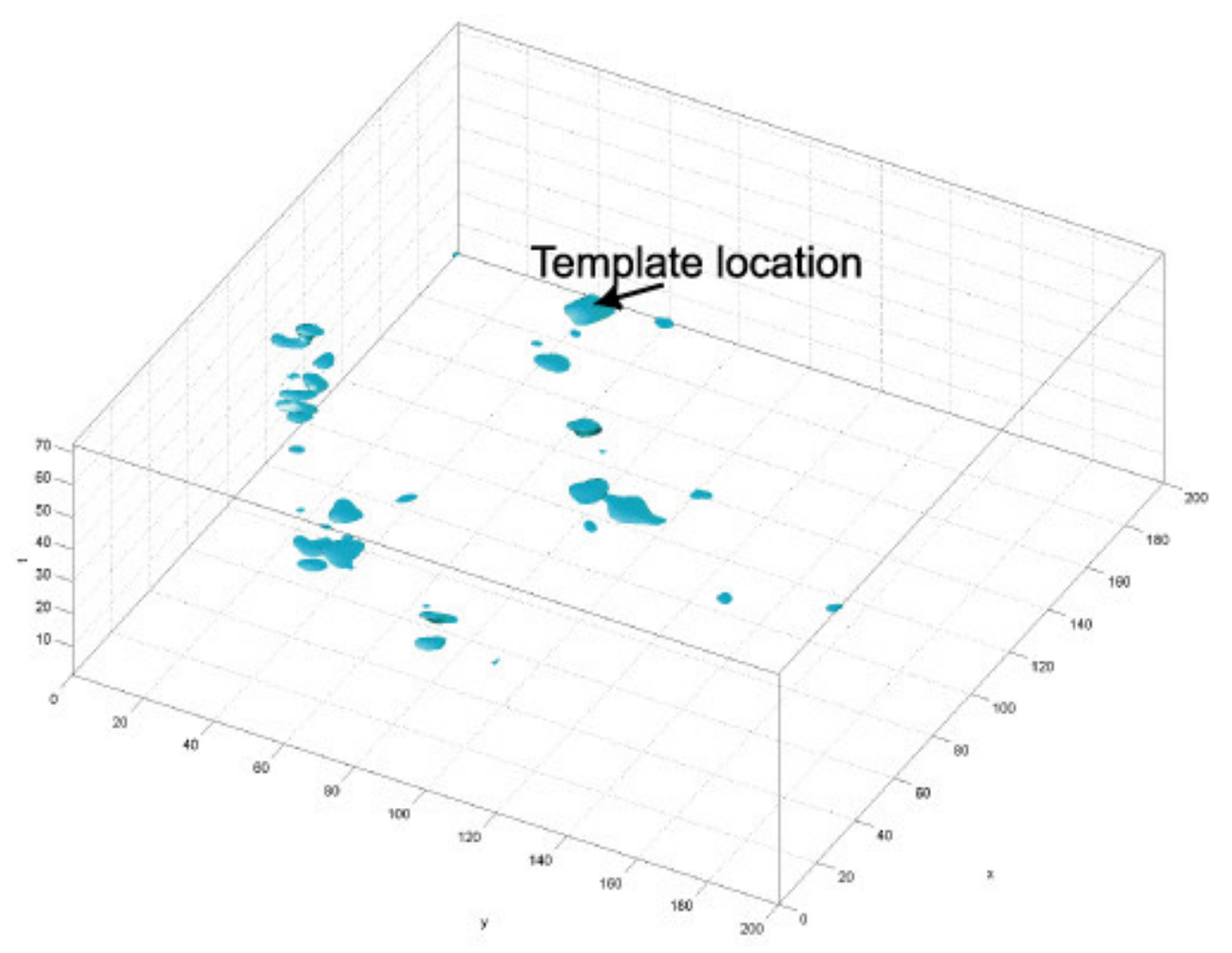

FIGURE 4. The movie seen as a single 3D data set was correlated with the kernel of Figure 3. Isosurfaces corresponding to a third of the maximum correlation value are rendered in blue. The position of the template is indicated.

\section{Decreasing false positive rates}

In order to reduce the number of candidate fusion events from 130 to a manageable number, we examine the value from the correlation volume of Figure 4, within the immediate neighborhood of each trajectory ending. A death is confirmed as a fusion event if the correlation value within a small $(7 \times 7 \times 6)$ neighborhood of the death event reaches the value used to define the iso-surface of Figure 4. This selection operation left only five events, shown row-wise in Figure 5. Four events out of five appear genuine and we could not visually identify additional events that the software had missed. Row four does not seem to correspond to a fusion event. Rather, a neighboring fusion event (the template event of row 5) seems to cause the disappearance of the vesicle.

\section{CONCLUSION}

We have developed and illustrated a new method for identifying fusion events in TIRFM movies of trafficking pancreatic $\beta$-cells. Our results obtained on a single movie suggest that the algorithm has a good ability to home in on fusion events. Further work will be required to investigate the ability of the algorithm to generalize. 
In particular, the use of multiple templates, and the use of a bootstrap method to improve the template construction will add more robustness to the method. As suggested by a reviewer, the use of rotationally symmetric kernels may also prove useful in that regard. In the near future we aim to acquire many more movies and to develop a truly high-throughput and validated method for their analysis.
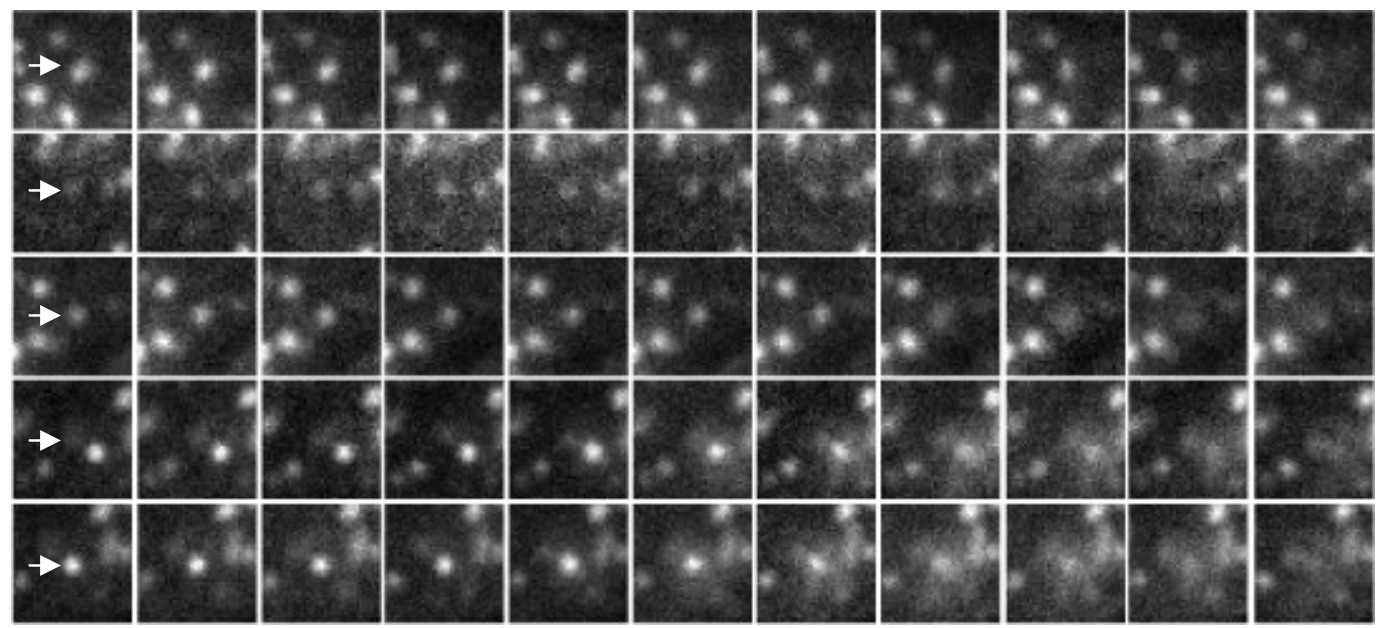

FIGURE 5. All four fusion events detected by the algorithm are shown in the four first rows of the table. Images were cropped such that the events occur in the very centre of the cropped images. Note in particular the second row, which would be difficult to detect visually. The fourth row is believed to be a false positive event. The last row corresponds to the area that we used as the template.

\section{ACKNOWLEDGMENTS}

We are grateful to David Mitchell and an anonymous reviewer for providing valuable comments on this manuscript.

\section{REFERENCES}

1. Schneckenburger $\mathrm{H}$. Total internal reflection fluorescence microscopy: Technical innovations and novel applications. Curr Opin Biotechnol. 2005;16(1):13-8.

2. Huang S, Lifshitz LM, Jones C, Bellve KD, Standley C, Fonseca S, Corvera S, Fogarty KE, Czech MP. Insulin stimulates membrane fusion and GLUT4 accumulation in clathrin coats on adipocyte plasma membranes. Mol Cell Biol. 2007;27(9):3456-69. Epub 2007 Mar 5.

3. Tran VS, Huet S, Fanget I, Cribier S, Henry JP, Karatekin E. Characterization of sequential exocytosis in a human neuroendocrine cell line using evanescent wave microscopy and "virtual trajectory" analysis. Eur Biophys J. 2007;18:18.

4. Hughes WE, Elgundi Z, Huang P, Frohman MA, Biden TJ. Phospholipase D1 regulates secretagoguestimulated insulin release in pancreatic beta-cells. J Biol Chem. 2004;279(26):27534-41. Epub 2004 Apr 14.

5. Vallotton P, Ponti A, Waterman-Storer CM, Salmon ED, Danuser G. Recovery, visualization, and analysis of actin and tubulin polymer flow in live cells: a fluorescent speckle microscopy study. Biophys J. 2003;85(2):1289-306. 